\title{
Oscillation Criteria of Third-Order Nonlinear Damped Dynamic Equations on Time Scales
}

\author{
Yang-Cong Qiu \\ School of Humanities and Social Science, Shunde Polytechnic, Foshan, Guangdong 528333, China \\ Correspondence should be addressed to Yang-Cong Qiu; q840410@qq.com
}

Received 4 February 2015; Accepted 31 March 2015

Academic Editor: Allan C. Peterson

Copyright (C) 2015 Yang-Cong Qiu. This is an open access article distributed under the Creative Commons Attribution License, which permits unrestricted use, distribution, and reproduction in any medium, provided the original work is properly cited.

We establish oscillation criteria of third-order nonlinear damped dynamic equations on time scales of the form $\left(r_{1}(t)\left(r_{2}(t)\left(x^{\Delta}(t)\right)^{\gamma}\right)^{\Delta}\right)^{\Delta}+f\left(t, x(t), x^{\sigma}(t), x(g(t)), x^{\Delta}(t)\right)=0$ by employing functions in some function classes and the generalized Riccati transformation. Two examples are given to show the significance of the conclusions.

\section{Introduction}

In this paper, we study third-order nonlinear damped dynamic equation

$$
\begin{aligned}
& \left(r_{1}(t)\left(r_{2}(t)\left(x^{\Delta}(t)\right)^{\gamma}\right)^{\Delta}\right)^{\Delta} \\
& \quad+f\left(t, x(t), x^{\sigma}(t), x(g(t)), x^{\Delta}(t)\right)=0
\end{aligned}
$$

on a time scale $\mathbb{T}$ satisfying inf $\mathbb{T}=t_{0}$ and $\sup \mathbb{\mathbb { T }}=\infty$.

Throughout this paper we shall assume that

(C1) $r_{1}, r_{2} \in C_{\mathrm{rd}}(\mathbb{T},(0, \infty))$ such that

$$
\int_{t_{0}}^{\infty} \frac{1}{r_{1}(t)} \Delta t=\infty, \quad \int_{t_{0}}^{\infty} \frac{1}{r_{2}^{1 / \gamma}(t)} \Delta t=\infty ;
$$

(C2) $\gamma$ is a quotient of odd positive integers;

(C3) $g \in C(\mathbb{T}, \mathbb{T})$, and for any $t \in \mathbb{T}$,

$$
g(t) \geq \begin{cases}\sigma(t), & 0<\gamma<1, \\ t, & \gamma \geq 1\end{cases}
$$

(C4) $f \in C\left(\mathbb{T} \times \mathbb{R}^{4}, \mathbb{R}\right)$ and there exists a function $q \in$ $C_{\mathrm{rd}}(\mathbb{T},(0, \infty))$ such that for $u, v, w$ with a same sign,

$$
f(t, u, v, w, r) \cdot \operatorname{sign}(u) \geq q(t)\left(|v|^{\gamma}+|w|^{\gamma}\right) ;
$$

(C5) when $0<\gamma<1$, it always satisfies

$$
\int_{t_{0}}^{\infty} q(t) \Delta t<\infty .
$$

In 1988, Hilger introduced the theory of time scales in his Ph.D. thesis [1] in order to unify continuous and discrete analysis; see also [2]. Preliminaries about time scale calculus can be found in [3-6] and omitted here.

Definition 1. A solution $x$ of (1) is said to have a generalized zero at $t^{*} \in \mathbb{T}$ if $x\left(t^{*}\right) x\left(\sigma\left(t^{*}\right)\right) \leq 0$, and it is said to be nonoscillatory on $\mathbb{T}$ if there exists $t_{0} \in \mathbb{T}$ such that $x(t) x(\sigma(t))$ $>0$ for all $t>t_{0}$. Otherwise, it is oscillatory. Equation (1) is said to be oscillatory if all solutions of (1) are oscillatory.

There has been much research achievement about the oscillation of dynamic equations on time scales in the last few years; see the papers [1-14] and the references therein.

Wang [14] discussed the even order nonlinear damped differential equation

$$
\begin{aligned}
x^{(n)}(t)+ & p(t) x^{(n-1)}(t) \\
+f( & t, x\left[\tau_{01}(t)\right], \ldots, x\left[\tau_{0 m}(t)\right], \ldots, \\
& \left.x^{(n-1)}\left[\tau_{n-11}(t)\right], \ldots, x^{(n-1)}\left[\tau_{n-1 m}(t)\right]\right)=0
\end{aligned}
$$


and obtained some oscillation criteria for (6). Hassan [8] gave some oscillation criteria for the third order nonlinear delay dynamic equation

$$
\left(a(t)\left[\left(r(t) x^{\Delta}(t)\right)^{\Delta}\right]^{\gamma}\right)^{\Delta}+f(t, x(\tau(t)))=0 .
$$

Afterwards, Erbe et al. [7] established some new oscillation criteria for (7). Saker et al. [11] studied the second-order damped dynamic equation

$$
\left(a(t) x^{\Delta}(t)\right)^{\Delta}+p(t) x^{\Delta^{\sigma}}(t)+q(t)\left(f \circ x^{\sigma}\right)=0
$$

and they gave some oscillation criteria.

Qiu and Wang [9] considered the second-order nonlinear dynamic equation

$$
\left(p(t) \psi(x(t)) k \circ x^{\Delta}(t)\right)^{\Delta}+f(t, x(\sigma(t)))=0 .
$$

By using a generalized Riccati transformation

$$
u(t)=A(t) \frac{p(t) \psi(x(t)) k \circ x^{\Delta}(t)}{x(t)}+B(t)
$$

the authors established some Kamenev-type oscillation criteria. Şenel [12] had tried to establish Kamenev-type oscillation criteria for the second-order nonlinear dynamic equation of the form

$$
\left(r(t)\left(x^{\Delta}(t)\right)^{\gamma}\right)^{\Delta}+p(t)\left(x^{\Delta}(t)\right)^{\gamma}+f(t, x(g(t)))=0 .
$$

However, it seemed that the obtained theorems and corollaries are incorrect. Qiu and Wang [10] corrected some mistakes in [12] and established correct oscillation criteria for (11) by employing functions in some function classes and the generalized Riccati transformation. Şenel [13] considered the third-order nonlinear dynamic equation

$$
\left(r_{1}(t)\left(r_{2}(t) x^{\Delta}(t)\right)^{\Delta}\right)^{\Delta}+P\left(t, x(t), x^{\Delta}(t)\right)+F(t, x(t))=0
$$

and established some sufficient conditions which guarantee that every solution of (12) oscillates or converges to zero on an arbitrary time scale $\mathbb{T}$. In this paper, we shall establish new oscillation criteria of (1), which is more general than (12), and give two examples to show the significance of the results.

For simplicity, throughout this paper, we denote $(a, b) \cap$ $\mathbb{T}=(a, b)_{\mathbb{T}}$, where $a, b \in \mathbb{R}$, and $[a, b]_{\mathbb{T}},[a, b)_{\mathbb{T}},(a, b]_{\mathbb{T}}$ are denoted similarly.

\section{Preliminary Results}

To establish oscillation criteria of (1), we give four lemmas in this section.

Lemma 2. Assume that (C1)-(C5) hold and there exists a sufficiently large $t_{1} \in\left[t_{0}, \infty\right)_{\mathbb{T}}$ such that $x(t)$ is a solution of (1) satisfying $x(t)>0$ for $t \in\left[t_{1}, \infty\right)_{\mathbb{T}}$. Then there exists $T \in$ $\left[t_{1}, \infty\right)_{\mathbb{V}}$ such that for $t \in[T, \infty)_{\mathbb{T}}$, we have

$$
\begin{gathered}
\left(r_{2}(t)\left(x^{\Delta}(t)\right)^{\gamma}\right)^{\Delta}>0, \\
x^{\Delta}(t)>0 \quad \text { or } \quad x^{\Delta}(t)<0 .
\end{gathered}
$$

Proof. Let $t_{1} \in\left[t_{0}, \infty\right)_{\mathbb{T}}$ such that $x(t)$ is a solution of (1) satisfying $x(t)>0$ for $t \in\left[t_{1}, \infty\right)_{\mathbb{T}}$; then we also have $x(\sigma(t))$, $x(g(t))>0$. By (1) and (C4), it follows that, for $t \in\left[t_{1}, \infty\right)_{\mathbb{T}}$,

$$
\begin{aligned}
& \left(r_{1}(t)\left(r_{2}(t)\left(x^{\Delta}(t)\right)^{\gamma}\right)^{\Delta}\right)^{\Delta} \\
& =-f\left(t, x(t), x^{\sigma}(t), x(g(t)), x^{\Delta}(t)\right) \\
& \leq-q(t)\left(x^{\gamma}(\sigma(t))+x^{\gamma}(g(t))\right)<0 .
\end{aligned}
$$

Hence, $r_{1}(t)\left(r_{2}(t)\left(x^{\Delta}(t)\right)^{\gamma}\right)^{\Delta}$ is strictly decreasing on $\left[t_{1}, \infty\right)_{\mathbb{T}}$. We claim that

$$
r_{1}(t)\left(r_{2}(t)\left(x^{\Delta}(t)\right)^{\gamma}\right)^{\Delta}>0, \quad t \in\left[t_{1}, \infty\right)_{\mathbb{T}}
$$

Assume not, then there exists $t_{2} \in\left[t_{1}, \infty\right)_{\mathbb{T}}$ such that $r_{1}(t)\left(r_{2}(t)\left(x^{\Delta}(t)\right)^{\gamma}\right)^{\Delta}<0$ for $t \in\left[t_{2}, \infty\right)_{\mathbb{T}}$. So there exists a constant $c<0$ and $t_{3} \in\left[t_{2}, \infty\right)_{\mathbb{T}}$ such that $r_{1}(t)\left(r_{2}(t)\left(x^{\Delta}(t)\right)^{\gamma}\right)^{\Delta} \leq c$ for $t \in\left[t_{3}, \infty\right)_{\mathbb{T}}$, which means that

$$
\left(r_{2}(t)\left(x^{\Delta}(t)\right)^{\gamma}\right)^{\Delta} \leq \frac{c}{r_{1}(t)}, \quad t \in\left[t_{3}, \infty\right)_{\mathbb{T}} .
$$

Integrating (16) from $t_{3}$ to $t \in\left[\sigma\left(t_{3}\right), \infty\right)_{\mathbb{T}}$, we obtain

$$
r_{2}(t)\left(x^{\Delta}(t)\right)^{\gamma} \leq r_{2}\left(t_{3}\right)\left(x^{\Delta}\left(t_{3}\right)\right)^{\gamma}+c \int_{t_{3}}^{t} \frac{\Delta s}{r_{1}(s)} .
$$

Letting $t \rightarrow \infty$, by (C1) we have $r_{2}(t)\left(x^{\Delta}(t)\right)^{\gamma} \rightarrow-\infty$. Then there exists $t_{4} \in\left[t_{3}, \infty\right)_{\mathbb{T}}$ such that $r_{2}(t)\left(x^{\Delta}(t)\right)^{\gamma} \leq$ $r_{2}\left(t_{4}\right)\left(x^{\Delta}\left(t_{4}\right)\right)^{\gamma}<0$ for $t \in\left[t_{4}, \infty\right)_{\mathbb{T}}$, which implies that

$$
x^{\Delta}(t) \leq r_{2}^{1 / \gamma}\left(t_{4}\right) x^{\Delta}\left(t_{4}\right) \cdot \frac{1}{r_{2}^{1 / \gamma}(t)} .
$$

Integrating (18) from $t_{4}$ to $t \in\left[\sigma\left(t_{4}\right), \infty\right)_{\mathbb{T}}$, we obtain

$$
x(t)-x\left(t_{4}\right) \leq r_{2}^{1 / \gamma}\left(t_{4}\right) x^{\Delta}\left(t_{4}\right) \int_{t_{4}}^{t} \frac{\Delta s}{r_{2}^{1 / \gamma}(s)} .
$$

Letting $t \rightarrow \infty$, by $(\mathrm{C} 1)$ we have $x(t) \rightarrow-\infty$, which contradicts $x(t)>0$. So (15) holds, which implies that

$$
\left(r_{2}(t)\left(x^{\Delta}(t)\right)^{\gamma}\right)^{\Delta}>0, \quad t \in\left[t_{1}, \infty\right)_{\mathbb{T}} .
$$

Therefore, $r_{2}(t)\left(x^{\Delta}(t)\right)^{\gamma}$ is strictly increasing on $\left[t_{1}, \infty\right)_{\mathbb{T}}$. It follows that $r_{2}(t)\left(x^{\Delta}(t)\right)^{\gamma}$ is either eventually positive or eventually negative. Then, there exists $T \in\left[t_{1}, \infty\right)_{\mathbb{T}}$ such that, for $t \in[T, \infty)_{\mathbb{T}}$, we have (13) holding. Lemma 2 is proved. 
Lemma 3. For $0<\gamma<1$, assume that (C1)-(C5) hold and $x(t)$ is a solution of (1) satisfying $x(t)>0, x^{\Delta}(t)>0$ for $t \in\left[t_{1}, \infty\right)_{\mathbb{W}}$ with $t_{1} \in\left[t_{0}, \infty\right)_{\mathbb{T}}$. Then, we have

$$
\begin{array}{r}
\left(\frac{x^{\Delta}(t)}{x^{\sigma}(t)}\right)^{1-\gamma} \geq \alpha(t)=\left(\frac{2 \delta(t)}{r_{2}(t)} \int_{t}^{\infty} q(s) \Delta s\right)^{(1-\gamma) / \gamma}, \\
t \in\left[t_{1}, \infty\right)_{\mathbb{T}},
\end{array}
$$

where

$$
\delta(t)=\int_{t_{1}}^{t} \frac{\Delta s}{r_{1}(s)} .
$$

Proof. Since $x(t)$ is a solution of (1) satisfying $x(t)>0$ for $t \in\left[t_{1}, \infty\right)_{\mathbb{T}}$ with $t_{1} \in\left[t_{0}, \infty\right)_{\mathbb{T}}$, by Lemma 2 we have

$$
\left(r_{2}(t)\left(x^{\Delta}(t)\right)^{\gamma}\right)^{\Delta}>0, \quad t \in\left[t_{1}, \infty\right)_{\mathbb{T}} .
$$

By $x^{\Delta}(t)>0$, it follows that, for $t \in\left[t_{1}, \infty\right)_{\mathbb{T}}$,

$$
\begin{aligned}
& \left(r_{1}(t)\left(r_{2}(t)\left(x^{\Delta}(t)\right)^{\gamma}\right)^{\Delta}\right)^{\Delta} \\
& \quad=-f\left(t, x(t), x^{\sigma}(t), x(g(t)), x^{\Delta}(t)\right) \\
& \quad \leq-q(t)\left(x^{\gamma}(\sigma(t))+x^{\gamma}(g(t))\right) \\
& \quad \leq-2 q(t) x^{\gamma}(\sigma(t)) .
\end{aligned}
$$

Integrating (24) from $\infty$ to $t \in\left[t_{1}, \infty\right)_{\mathbb{T}}$, by (23) we obtain

$$
\begin{aligned}
r_{1}(t) & \left(r_{2}(t)\left(x^{\Delta}(t)\right)^{\gamma}\right)^{\Delta} \\
\geq & \int_{\infty}^{t}\left(r_{1}(s)\left(r_{2}(s)\left(x^{\Delta}(s)\right)^{\gamma}\right)^{\Delta}\right)^{\Delta} \Delta s \\
= & -\int_{t}^{\infty}\left(r_{1}(s)\left(r_{2}(s)\left(x^{\Delta}(s)\right)^{\gamma}\right)^{\Delta}\right)^{\Delta} \Delta s \\
\geq & \int_{t}^{\infty} 2 q(s) x^{\gamma}(\sigma(s)) \Delta s \geq 2 x^{\gamma}(\sigma(t)) \int_{t}^{\infty} q(s) \Delta s .
\end{aligned}
$$

As $r_{1}(t)\left(r_{2}(t)\left(x^{\Delta}(t)\right)^{\gamma}\right)^{\Delta}$ is strictly decreasing on $\left[t_{1}, \infty\right)_{\mathbb{T}}$, for $t \in\left[\sigma\left(t_{1}\right), \infty\right)_{\mathbb{W}}$ we have

$$
\begin{aligned}
& r_{2}(t)\left(x^{\Delta}(t)\right)^{\gamma} \\
&= r_{2}\left(t_{1}\right)\left(x^{\Delta}\left(t_{1}\right)\right)^{\gamma} \\
& \quad+\int_{t_{1}}^{t} \frac{r_{1}(s)\left(r_{2}(s)\left(x^{\Delta}(s)\right)^{\gamma}\right)^{\Delta}}{r_{1}(s)} \Delta s \\
& \geq r_{1}(t)\left(r_{2}(t)\left(x^{\Delta}(t)\right)^{\gamma}\right)^{\Delta} \int_{t_{1}}^{t} \frac{1}{r_{1}(s)} \Delta s \\
& \geq 2 x^{\gamma}(\sigma(t)) \delta(t) \int_{t}^{\infty} q(s) \Delta s .
\end{aligned}
$$

Hence when $0<\gamma<1$, we have

$$
\left(\frac{x^{\Delta}(t)}{x^{\sigma}(t)}\right)^{\gamma} \geq \frac{2 \delta(t)}{r_{2}(t)} \int_{t}^{\infty} q(s) \Delta s, \quad t \in\left[t_{1}, \infty\right)_{\mathbb{T}},
$$

which implies that

$$
\left(\frac{x^{\Delta}(t)}{x^{\sigma}(t)}\right)^{1-\gamma} \geq \alpha(t), \quad t \in\left[t_{1}, \infty\right)_{\mathbb{T}} .
$$

Lemma 3 is proved.

Lemma 4. For $\gamma \geq 1$, assume that (C1)-(C4) hold and $x(t)$ is a solution of (1) satisfying $x(t)>0, x^{\Delta}(t)<0$ for $t \in\left[t_{1}, \infty\right)_{\mathbb{T}}$ with $t_{1} \in\left[t_{0}, \infty\right)_{\mathbb{T}}$, and

$$
\int_{t_{0}}^{\infty} q(t) \Delta t=\infty .
$$

Then $\lim _{t \rightarrow \infty} x(t)=0$.

Proof. Since $x(t)$ is a solution of (1) satisfying $x(t)>0$ for $t \in\left[t_{1}, \infty\right)_{\mathbb{T}}$ with $t_{1} \in\left[t_{0}, \infty\right)_{\mathbb{T}}$, by Lemma 2 we have

$$
\left(r_{2}(t)\left(x^{\Delta}(t)\right)^{\gamma}\right)^{\Delta}>0, \quad t \in\left[t_{1}, \infty\right)_{\mathbb{V}} .
$$

By $x^{\Delta}(t)<0$, there exists $b \geq 0$ such that $\lim _{t \rightarrow \infty} x(t)=b$. Assume $b>0$, by (24) and $x(\sigma(t)), x(g(t))>b$, we obtain

$$
\begin{aligned}
& \left(r_{1}(t)\left(r_{2}(t)\left(x^{\Delta}(t)\right)^{\gamma}\right)^{\Delta}\right)^{\Delta} \\
& \quad \leq-q(t)\left(x^{\gamma}(\sigma(t))+x^{\gamma}(g(t))\right)<-2 b^{\gamma} q(t) .
\end{aligned}
$$

Letting $v(t)=r_{1}(t)\left(r_{2}(t)\left(x^{\Delta}(t)\right)^{\gamma}\right)^{\Delta}, t \in\left[t_{1}, \infty\right)_{\mathbb{T}}$, we have $v(t)>0$, and

$$
v^{\Delta}(t)<-2 b^{\gamma} q(t), \quad t \in\left[t_{1}, \infty\right)_{\mathbb{T}} .
$$

Integrating (32) from $t_{1}$ to $t \in\left[\sigma\left(t_{1}\right), \infty\right)_{\mathbb{T}}$, we obtain

$$
v(t)<v\left(t_{1}\right)-2 b^{\gamma} \int_{t_{1}}^{t} q(s) \Delta s .
$$

By (29), there exists a sufficiently large $t_{2} \in\left[t_{1}, \infty\right)_{\mathbb{T}}$ such that $v(t)<0, t \in\left[t_{2}, \infty\right)_{\mathbb{T}}$, which contradicts $v(t)>0$. So $b=0$. Lemma 4 is proved.

Lemma 5. Assume that (C1)-(C5) hold and $x(t)$ is a solution of (1) satisfying $x(t)>0, x^{\Delta}(t)>0$ for $t \in\left[t_{1}, \infty\right)_{\mathbb{W}}$ with $t_{1} \in\left[t_{0}, \infty\right)_{\mathbb{T}}$. For $t \in\left[t_{1}, \infty\right)_{\mathbb{T}}$, define

$$
u(t)=A(t) \frac{r_{1}(t)\left(r_{2}(t)\left(x^{\Delta}(t)\right)^{\gamma}\right)^{\Delta}}{x^{\gamma}(t)}+B(t),
$$

where $A \in C_{\mathrm{rd}}^{1}(\mathbb{T},(0, \infty))$ and $B \in C_{\mathrm{rd}}^{1}(\mathbb{T}, \mathbb{R})$. Then, $u(t)$ satisfies

$$
u^{\Delta}(t)+2 A(t) q(t)-B^{\Delta}(t)-\Phi_{0}(t) \leq 0,
$$


where

$$
\begin{aligned}
& \Phi_{0}(t) \\
& =\left\{\begin{array}{c}
A^{\Delta}(t)\left(\frac{u(t)-B(t)}{A(t)}\right)^{\sigma} \\
-\frac{\gamma A(t) \delta(t) \alpha(t)}{r_{2}(t)}\left[\left(\frac{u(t)-B(t)}{A(t)}\right)^{\sigma}\right]^{2}, \\
0<\gamma<1, \\
A^{\Delta}(t)\left(\frac{u(t)-B(t)}{A(t)}\right)^{\sigma} \\
-\gamma A(t)\left(\frac{\delta(t)}{r_{2}(t)}\right)^{1 / \gamma}\left[\left(\frac{u(t)-B(t)}{A(t)}\right)^{\sigma}\right]^{(1+\gamma) / \gamma}, \\
\gamma \geq 1 .
\end{array}\right.
\end{aligned}
$$

Proof. Without loss of generality we may assume there exists $t_{1} \in\left[t_{0}, \infty\right)_{\mathbb{W}}$ such that $x(t)>0, x^{\Delta}(t)>0$ for $t \in\left[t_{1}, \infty\right)_{\mathbb{J}}$, then Lemmas 2 and 3 hold. Let $u(t)$ be defined by (34). Then, differentiating (34) and using (1), it follows that

$$
\begin{aligned}
u^{\Delta}= & \left(\frac{A}{x^{\gamma}}\right)\left(r_{1}\left(r_{2}\left(x^{\Delta}\right)^{\gamma}\right)^{\Delta}\right)^{\Delta} \\
& +\left(\frac{A}{x^{\gamma}}\right)^{\Delta}\left(r_{1}\left(r_{2}\left(x^{\Delta}\right)^{\gamma}\right)^{\Delta}\right)^{\sigma}+B^{\Delta} \\
= & \left(\frac{A}{x^{\gamma}}\right)\left(-f\left(t, x(t), x^{\sigma}(t), x(g(t)), x^{\Delta}(t)\right)\right) \\
& +\frac{A^{\Delta} x^{\gamma}-A\left(x^{\gamma}\right)^{\Delta}}{x^{\gamma}\left(x^{\gamma}\right)^{\sigma}}\left(r_{1}\left(r_{2}\left(x^{\Delta}\right)^{\gamma}\right)^{\Delta}\right)^{\sigma}+B^{\Delta} .
\end{aligned}
$$

Using the fact that

$$
\begin{aligned}
& f\left(t, x(t), x^{\sigma}(t), x(g(t)), x^{\Delta}(t)\right) \\
& \quad \geq q(t)\left(x^{\gamma}(\sigma(t))+x^{\gamma}(g(t))\right) \geq 2 q(t) x^{\gamma}(t),
\end{aligned}
$$

we obtain

$$
u^{\Delta} \leq-2 A q+B^{\Delta}+A^{\Delta}\left(\frac{u-B}{A}\right)^{\sigma}-A \frac{\left(x^{\gamma}\right)^{\Delta}}{x^{\gamma}}\left(\frac{u-B}{A}\right)^{\sigma} .
$$

When $0<\gamma<1$, using the Pötzsche chain rule, we have

$$
\left(x^{\gamma}\right)^{\Delta}=\gamma \int_{0}^{1}\left(x+h \mu x^{\Delta}\right)^{\gamma-1} d h \cdot x^{\Delta} \geq \gamma\left(x^{\sigma}\right)^{\gamma-1} x^{\Delta},
$$

and it follows that

$$
\frac{\left(x^{\gamma}\right)^{\Delta}}{x^{\gamma}} \geq \frac{\gamma\left(x^{\sigma}\right)^{\gamma-1} x^{\Delta}}{x^{\gamma}}=\gamma \frac{x^{\Delta}}{x^{\sigma}}\left(\frac{x^{\sigma}}{x}\right)^{\gamma} .
$$

By Lemmas 2 and 3 , for $t \in\left[t_{1}, \infty\right)_{\mathbb{T}}$, we obtain

$$
\begin{gathered}
\frac{x^{\Delta}}{x^{\sigma}}=\frac{1}{r_{2}} \frac{r_{2}\left(x^{\Delta}\right)^{\gamma}}{\left(x^{\sigma}\right)^{\gamma}}\left(\frac{x^{\Delta}}{x^{\sigma}}\right)^{1-\gamma} \geq \frac{\delta \alpha}{r_{2}} \frac{r_{1}\left(r_{2}\left(x^{\Delta}\right)^{\gamma}\right)^{\Delta}}{\left(x^{\gamma}\right)^{\sigma}} \\
\geq \frac{\delta \alpha}{r_{2}} \frac{\left(r_{1}\left(r_{2}\left(x^{\Delta}\right)^{\gamma}\right)^{\Delta}\right)^{\sigma}}{\left(x^{\gamma}\right)^{\sigma}}=\frac{\delta \alpha}{r_{2}}\left(\frac{u-B}{A}\right)^{\sigma}, \\
\frac{x^{\sigma}}{x} \geq 1 .
\end{gathered}
$$

So (39) becomes

$$
u^{\Delta} \leq-2 A q+B^{\Delta}+A^{\Delta}\left(\frac{u-B}{A}\right)^{\sigma}-\frac{\gamma A \delta \alpha}{r_{2}}\left[\left(\frac{u-B}{A}\right)^{\sigma}\right]^{2} .
$$

When $\gamma \geq 1$, we have

$$
\left(x^{\gamma}\right)^{\Delta}=\gamma \int_{0}^{1}\left(x+h \mu x^{\Delta}\right)^{\gamma-1} d h \cdot x^{\Delta} \geq \gamma x^{\gamma-1} x^{\Delta},
$$

and it follows that

$$
\frac{\left(x^{\gamma}\right)^{\Delta}}{x^{\gamma}} \geq \frac{\gamma x^{\gamma-1} x^{\Delta}}{x^{\gamma}}=\frac{\gamma x^{\Delta}}{x} .
$$

By Lemmas 2 and 3 , for $t \in\left[t_{1}, \infty\right)_{\mathbb{T}}$, we obtain

$$
\begin{aligned}
\left(\frac{x^{\Delta}}{x}\right)^{\gamma} & =\frac{1}{r_{2}} \frac{r_{2}\left(x^{\Delta}\right)^{\gamma}}{x^{\gamma}} \geq \frac{\delta}{r_{2}} \frac{r_{1}\left(r_{2}\left(x^{\Delta}\right)^{\gamma}\right)^{\Delta}}{x^{\gamma}} \\
& \geq \frac{\delta}{r_{2}} \frac{\left(r_{1}\left(r_{2}\left(x^{\Delta}\right)^{\gamma}\right)^{\Delta}\right)^{\sigma}}{\left(x^{\gamma}\right)^{\sigma}}=\frac{\delta}{r_{2}}\left(\frac{u-B}{A}\right)^{\sigma},
\end{aligned}
$$

which implies that

$$
\frac{x^{\Delta}}{x} \geq\left(\frac{\delta}{r_{2}}\right)^{1 / \gamma}\left[\left(\frac{u-B}{A}\right)^{\sigma}\right]^{1 / \gamma} .
$$

So (39) becomes

$$
\begin{aligned}
u^{\Delta} \leq & -2 A q+B^{\Delta}+A^{\Delta}\left(\frac{u-B}{A}\right)^{\sigma} \\
& -\gamma A\left(\frac{\delta}{r_{2}}\right)^{1 / \gamma}\left[\left(\frac{u-B}{A}\right)^{\sigma}\right]^{(1+\gamma) / \gamma} .
\end{aligned}
$$

By (43) and (48), (35) holds. Lemma 5 is proved.

\section{Main Results}

In this section, we establish oscillation criteria of (1) by generalized Riccati transformation. Firstly, we give some definitions. 
Let $D_{0}=\{s \in \mathbb{T}: s \geq 0\}$ and $D=\left\{(t, s) \in \mathbb{T}^{2}: t \geq s \geq 0\right\}$. For any function $f(t, s): \mathbb{T}^{2} \rightarrow \mathbb{R}$, denote by $f_{2}^{\Delta}$ the partial derivative of $f$ with respect to $s$. Define

$$
\begin{aligned}
& (\mathscr{A}, \mathscr{B}) \\
& =\left\{(A, B): A(s) \in C_{\mathrm{rd}}^{1}\left(D_{0},(0, \infty)\right),\right. \\
& \left.\quad B(s) \in C_{\mathrm{rd}}^{1}\left(D_{0}, \mathbb{R}\right), s \in D_{0}\right\} ; \\
& \mathscr{H}=\left\{H(t, s) \in C^{1}(D,[0, \infty)): H(t, t)=0,\right. \\
& \left.\quad H(t, s)>0, H_{2}^{\Delta}(t, s) \leq 0, t>s \geq 0\right\} .
\end{aligned}
$$

These function classes will be used throughout this paper. Now, we give our first theorem.

Theorem 6. Assume that (C1)-(C5) hold and that there exist $(A, B) \in(\mathscr{A}, \mathscr{B})$ and $H \in \mathscr{H}$ such that, for any $t_{1} \in \mathbb{T}$,

$$
\begin{aligned}
\limsup _{t \rightarrow \infty} \frac{1}{H\left(t, t_{1}\right)} \int_{t_{1}}^{t}[ & H(t, s)\left(2 A(s) q(s)-B^{\Delta}(s)\right) \\
& \left.-H_{2}^{\Delta}(t, s) B^{\sigma}(s)-\Phi_{1}(s)\right] \Delta s=\infty,
\end{aligned}
$$

where

$$
\Phi_{1}(s)=\left\{\begin{array}{c}
\frac{r_{2}(s)\left(H_{2}^{\Delta}(t, s) A^{\sigma}(s)+H(t, s) A^{\Delta}(s)\right)^{2}}{4 \gamma H(t, s) A(s) \delta(s) \alpha(s)}, \\
0<\gamma<1, \\
\frac{r_{2}(s)}{\delta(s)(H(t, s) A(s))^{\gamma}} \\
\cdot\left(\frac{H_{2}^{\Delta}(t, s) A^{\sigma}(s)+H(t, s) A^{\Delta}(s)}{1+\gamma}\right)^{1+\gamma}, \\
\gamma \geq 1 .
\end{array}\right.
$$

Then, (1) is oscillatory or $\lim _{t \rightarrow \infty} x(t)$ exists.

Proof. Assume that (1) is not oscillatory. Without loss of generality we may assume there exists $t_{1} \in\left[t_{0}, \infty\right)_{\mathbb{T}}$ such that $x(t)>0$ for $t \in\left[t_{1}, \infty\right)_{\mathbb{T}}$. By Lemma 2 , for $t \in\left[t_{1}, \infty\right)_{\mathbb{T}}$, either $x^{\Delta}(t)>0$ or $x^{\Delta}(t)<0$ holds. Assume $x^{\Delta}(t)>0, t \in\left[t_{1}, \infty\right)_{\mathbb{T}}$. Let $u(t)$ be defined by (34). Then by Lemma 5 , (35) holds.

For simplicity in the following, we let $H=H(t, s), H_{2}^{\Delta}=$ $H_{2}^{\Delta}(t, s)$, and we omit the arguments in the integrals.

Multiplying (35), where $t$ is replaced by $s$, by $H$ and integrating it with respect to $s$ from $t_{1}$ to $t$ with $t \in\left[\sigma\left(t_{1}\right)\right.$, $\infty)_{\mathbb{T}}$, we obtain

$$
\int_{t_{1}}^{t} H\left(2 A q-B^{\Delta}\right) \Delta s \leq-\int_{t_{1}}^{t} H u^{\Delta} \Delta s+\int_{t_{1}}^{t} H \Phi_{0} \Delta s .
$$

Noting that $H(t, t)=0$, by the integration by parts formula we have

$$
\begin{aligned}
\int_{t_{1}}^{t} H\left(2 A q-B^{\Delta}\right) \Delta s \\
\leq H\left(t, t_{1}\right) u\left(t_{1}\right)+\int_{t_{1}}^{t}\left(H_{2}^{\Delta} u^{\sigma}+H \Phi_{0}\right) \Delta s \\
=H\left(t, t_{1}\right) u\left(t_{1}\right)+\int_{t_{1}}^{t} H_{2}^{\Delta} B^{\sigma} \Delta s \\
\quad+\int_{t_{1}}^{t}\left(H_{2}^{\Delta} A^{\sigma}\left(\frac{u-B}{A}\right)^{\sigma}+H \Phi_{0}\right) \Delta s .
\end{aligned}
$$

When $0<\gamma<1$, we have

$$
\begin{aligned}
H_{2}^{\Delta} A^{\sigma}\left(\frac{u-B}{A}\right)^{\sigma}+H \Phi_{0} \\
=\left(H_{2}^{\Delta} A^{\sigma}+H A^{\Delta}\right)\left(\frac{u-B}{A}\right)^{\sigma}-\frac{\gamma H A \delta \alpha}{r_{2}}\left[\left(\frac{u-B}{A}\right)^{\sigma}\right]^{2} \\
=\frac{r_{2}\left(H_{2}^{\Delta} A^{\sigma}+H A^{\Delta}\right)^{2}}{4 \gamma H A \delta \alpha}-\frac{\gamma H A \delta \alpha}{r_{2}} \\
\quad \cdot\left[\left(\frac{u-B}{A}\right)^{\sigma}-\frac{r_{2}\left(H_{2}^{\Delta} A^{\sigma}+H A^{\Delta}\right)}{2 \gamma H A \delta \alpha}\right]^{2} \\
\leq \frac{r_{2}\left(H_{2}^{\Delta} A^{\sigma}+H A^{\Delta}\right)^{2}}{4 \gamma H A \delta \alpha} .
\end{aligned}
$$

When $\gamma \geq 1$, we have

$$
\begin{aligned}
H_{2}^{\Delta} & A^{\sigma}\left(\frac{u-B}{A}\right)^{\sigma}+H \Phi_{0} \\
= & \left(H_{2}^{\Delta} A^{\sigma}+H A^{\Delta}\right)\left(\frac{u-B}{A}\right)^{\sigma} \\
& -\gamma H A\left(\frac{\delta}{r_{2}}\right)^{1 / \gamma}\left[\left(\frac{u-B}{A}\right)^{\sigma}\right]^{(1+\gamma) / \gamma} .
\end{aligned}
$$

Using the inequality

$$
\lambda a b^{\lambda-1}-a^{\lambda} \leq(\lambda-1) b^{\lambda},
$$

let $\lambda=(1+\gamma) / \gamma$, and

$$
\begin{aligned}
& a^{\lambda}=a^{(1+\gamma) / \gamma}=\gamma H A\left(\frac{\delta}{r_{2}}\right)^{1 / \gamma}\left[\left(\frac{u-B}{A}\right)^{\sigma}\right]^{(1+\gamma) / \gamma}, \\
& b^{\lambda-1}=b^{1 / \gamma}=\frac{\gamma}{1+\gamma}\left(\frac{r_{2}}{\delta}\right)^{1 /(1+\gamma)} \frac{H_{2}^{\Delta} A^{\sigma}+H A^{\Delta}}{(\gamma H A)^{\gamma /(1+\gamma)}},
\end{aligned}
$$

then we have

$$
H_{2}^{\Delta} A^{\sigma}\left(\frac{u-B}{A}\right)^{\sigma}+H \Phi_{0} \leq \frac{r_{2}}{\delta(H A)^{\gamma}}\left(\frac{H_{2}^{\Delta} A^{\sigma}+H A^{\Delta}}{1+\gamma}\right)^{1+\gamma} .
$$


Therefore, for all $\gamma>0$, by (53) we have

$$
\begin{aligned}
\int_{t_{1}}^{t} H\left(2 A q-B^{\Delta}\right) \Delta s \leq & H\left(t, t_{1}\right) u\left(t_{1}\right)+\int_{t_{1}}^{t} H_{2}^{\Delta} B^{\sigma} \Delta s \\
& +\int_{t_{1}}^{t} \Phi_{1} \Delta s,
\end{aligned}
$$

which implies that

$$
\int_{t_{1}}^{t}\left[H\left(2 A q-B^{\Delta}\right)-H_{2}^{\Delta} B^{\sigma}-\Phi_{1}\right] \Delta s \leq H\left(t, t_{1}\right) u\left(t_{1}\right) .
$$

Hence,

$\frac{1}{H\left(t, t_{1}\right)} \int_{t_{1}}^{t}\left[H\left(2 A q-B^{\Delta}\right)-H_{2}^{\Delta} B^{\sigma}-\Phi_{1}\right] \Delta s \leq u\left(t_{1}\right)<\infty$,

which contradicts $(50)$. So $x^{\Delta}(t)<0, t \in\left[t_{1}, \infty\right)_{\mathbb{T}}$, and it is clearly that $\lim _{t \rightarrow \infty} x(t)$ exists. The proof is completed.

When $\gamma \geq 1$, if (29) holds, we have the following corollary on the basis of Lemma 4 and Theorem 6 .

Corollary 7. When $\gamma \geq 1$, assume that (C1)-(C4) and (29) hold. If there exist $(A, B) \in(\mathscr{A}, \mathscr{B})$ and $H \in \mathscr{H}$ such that, for any $t_{1} \in \mathbb{T}$,

$$
\begin{aligned}
\limsup _{t \rightarrow \infty} \frac{1}{H\left(t, t_{1}\right)} \int_{t_{1}}^{t}\left[H(t, s)\left(2 A(s) q(s)-B^{\Delta}(s)\right)\right. \\
\left.\quad-H_{2}^{\Delta}(t, s) B^{\sigma}(s)-\Phi_{1}(s)\right] \Delta s=\infty,
\end{aligned}
$$

where

$$
\begin{aligned}
\Phi_{1}(s)= & \frac{r_{2}(s)}{\delta(s)(H(t, s) A(s))^{\gamma}} \\
& \cdot\left(\frac{H_{2}^{\Delta}(t, s) A^{\sigma}(s)+H(t, s) A^{\Delta}(s)}{1+\gamma}\right)^{1+\gamma} .
\end{aligned}
$$

Then, (1) is oscillatory or $\lim _{t \rightarrow \infty} x(t)=0$.

Remark 8. In Corollary 7, letting $(A, B)=(1,0)$, we can simplify (62) as

$$
\begin{aligned}
\limsup _{t \rightarrow \infty} & \frac{1}{H\left(t, t_{1}\right)} \\
\cdot \int_{t_{1}}^{t}[2 H(t, s) q(s) & {\left[\frac{r_{2}(s)}{\delta(s) H^{\gamma}(t, s)}\left(\frac{H_{2}^{\Delta}(t, s)}{1+\gamma}\right)^{1+\gamma}\right] \Delta s=\infty . }
\end{aligned}
$$

When $B=0$, (34) is simplified as

$$
u(t)=A(t) \frac{r_{1}(t)\left(r_{2}(t)\left(x^{\Delta}(t)\right)^{\gamma}\right)^{\Delta}}{x^{\gamma}(t)}, \quad t \in\left[t_{1}, \infty\right)_{\mathbb{T}} .
$$

Now we have the following theorem.

Theorem 9. Assume that (C1)-(C5) hold and that there exists $A \in C_{\mathrm{rd}}^{1}\left(D_{0},(0, \infty)\right)$ such that, for any $t_{1} \in \mathbb{T}$,

$$
\limsup _{t \rightarrow \infty} \int_{t_{1}}^{t}\left[2 A(s) q(s)-\Phi_{2}(s)\right] \Delta s=\infty,
$$

where

$$
\Phi_{2}(s)= \begin{cases}\frac{r_{2}(s)\left(A^{\Delta}(s)\right)^{2}}{4 \gamma A(s) \delta(s) \alpha(s)}, & 0<\gamma<1, \\ \frac{r_{2}(s)}{\delta(s) A^{\gamma}(s)}\left(\frac{A^{\Delta}(s)}{1+\gamma}\right)^{1+\gamma}, & \gamma \geq 1 .\end{cases}
$$

Then, (1) is oscillatory or $\lim _{t \rightarrow \infty} x(t)$ exists.

Proof. Assume that (1) is not oscillatory. Without loss of generality we may assume that there exists $t_{1} \in\left[t_{0}, \infty\right)_{\mathbb{T}}$ such that $x(t)>0$ for $t \in\left[t_{1}, \infty\right)_{\mathbb{T}}$. By Lemma 2, for $t \in\left[t_{1}, \infty\right)_{\mathbb{T}}$, either $x^{\Delta}(t)>0$ or $x^{\Delta}(t)<0$ holds. Assume $x^{\Delta}(t)>0$, $t \in\left[t_{1}, \infty\right)_{\mathbb{T}}$. Let $u(t)$ be defined by (65). Then by Lemma 5 , we have

$$
u^{\Delta}(t)+2 A(t) q(t)-\Phi_{0}(t) \leq 0,
$$

where $\Phi_{0}(t)$ is simplified as

$\Phi_{0}(t)$

$$
=\left\{\begin{array}{l}
A^{\Delta}(t)\left(\frac{u(t)}{A(t)}\right)^{\sigma}-\frac{\gamma A(t) \delta(t) \alpha(t)}{r_{2}(t)}\left[\left(\frac{u(t)}{A(t)}\right)^{\sigma}\right]^{2}, \\
0<\gamma<1, \\
A^{\Delta}(t)\left(\frac{u(t)}{A(t)}\right)^{\sigma} \\
\quad-\gamma A(t)\left(\frac{\delta(t)}{r_{2}(t)}\right)^{1 / \gamma}\left[\left(\frac{u(t)}{A(t)}\right)^{\sigma}\right]^{(1+\gamma) / \gamma}, \quad \gamma \geq 1 .
\end{array}\right.
$$

When $0<\gamma<1$, we have

$$
\begin{aligned}
u^{\Delta} & \leq-2 A q+A^{\Delta}\left(\frac{u}{A}\right)^{\sigma}-\frac{\gamma A \delta \alpha}{r_{2}}\left[\left(\frac{u}{A}\right)^{\sigma}\right]^{2} \\
& =-2 A q+\frac{r_{2}\left(A^{\Delta}\right)^{2}}{4 \gamma A \delta \alpha}-\frac{\gamma A \delta \alpha}{r_{2}}\left[\left(\frac{u}{A}\right)^{\sigma}-\frac{r_{2} A^{\Delta}}{2 \gamma A \delta \alpha}\right]^{2} \\
& \leq-2 A q+\frac{r_{2}\left(A^{\Delta}\right)^{2}}{4 \gamma A \delta \alpha} .
\end{aligned}
$$

When $\gamma \geq 1$, we have

$$
u^{\Delta} \leq-2 A q+A^{\Delta}\left(\frac{u}{A}\right)^{\sigma}-\gamma A\left(\frac{\delta}{r_{2}}\right)^{1 / \gamma}\left[\left(\frac{u}{A}\right)^{\sigma}\right]^{(1+\gamma) / \gamma} \text {. }
$$


Using the inequality

$$
\lambda a b^{\lambda-1}-a^{\lambda} \leq(\lambda-1) b^{\lambda}
$$

let $\lambda=(1+\gamma) / \gamma$, and

$$
\begin{aligned}
& a^{\lambda}=a^{(1+\gamma) / \gamma}=\gamma A\left(\frac{\delta}{r_{2}}\right)^{1 / \gamma}\left[\left(\frac{u}{A}\right)^{\sigma}\right]^{(1+\gamma) / \gamma}, \\
& b^{\lambda-1}=b^{1 / \gamma}=\frac{\gamma}{1+\gamma}\left(\frac{r_{2}}{\delta}\right)^{1 /(1+\gamma)} \frac{A^{\Delta}}{(\gamma A)^{\gamma /(1+\gamma)}},
\end{aligned}
$$

then we have

$$
u^{\Delta} \leq-2 A q+\frac{r_{2}}{\delta A^{\gamma}}\left(\frac{A^{\Delta}}{1+\gamma}\right)^{1+\gamma}
$$

Therefore, for all $\gamma>0$, we always have

$$
u^{\Delta} \leq-2 A q+\Phi_{2}
$$

which implies that

$$
2 A(t) q(t)-\Phi_{2}(t) \leq-u^{\Delta}(t) .
$$

Letting $t$ be replaced by $s$, and integrating (76) with respect to $s$ from $t_{1}$ to $t \in\left[\sigma\left(t_{1}\right), \infty\right)_{\mathbb{T}}$, we obtain

$$
\begin{aligned}
& \int_{t_{1}}^{t}\left[2 A(s) q(s)-\Phi_{2}(s)\right] \Delta s \\
& \quad \leq-\int_{t_{1}}^{t} u^{\Delta}(s) \Delta s=u\left(t_{1}\right)-u(t)<u\left(t_{1}\right)<\infty
\end{aligned}
$$

which is a contradiction of (66). So $x^{\Delta}(t)<0, t \in\left[t_{1}, \infty\right)_{\mathbb{T}}$, and, as before, $\lim _{t \rightarrow \infty} x(t)$ exists. The proof is completed.

When $\gamma \geq 1$, if (29) holds, from Lemma 4 and Theorem 9, we have the following result.

Corollary 10. When $\gamma \geq 1$, assume that (C1)-(C4) and (29) hold. If there exists $A \in C_{\mathrm{rd}}^{1}\left(D_{0},(0, \infty)\right)$ such that, for any $t_{1} \in$ ए,

$$
\begin{aligned}
& \limsup _{t \rightarrow \infty} \int_{t_{1}}^{t}\left[2 A(s) q(s)-\frac{r_{2}(s)}{\delta(s) A^{\gamma}(s)}\left(\frac{A^{\Delta}(s)}{1+\gamma}\right)^{1+\gamma}\right] \Delta s \\
& \quad=\infty
\end{aligned}
$$

then (1) is oscillatory or $\lim _{t \rightarrow \infty} x(t)=0$.

Remark 11. Actually, letting $A=1$, by (29) we have (78). Hence, it is easy to satisfy the conditions in Corollary 10.

Remark 12. When $\gamma \geq 1$, for $u, v, w$ with a same sign, if we assume that

$$
f(t, u, v, w, r) \cdot \operatorname{sign}(u) \geq q(t)\left(|u|^{\gamma}+|w|^{\gamma}\right)
$$

or

$$
f(t, u, v, w, r) \cdot \operatorname{sign}(u) \geq q(t)\left(|u|^{\gamma}+|v|^{\gamma}\right)
$$

instead of (4) in (C4), by (31) in Lemma 4, the conclusions above are also applicable. Furthermore, if $\gamma=1, g(t)=t$, and

$$
\begin{aligned}
& f\left(t, x(t), x^{\sigma}(t), x(g(t)), x^{\Delta}(t)\right) \\
& \quad=P\left(t, x(t), x^{\Delta}(t)\right)+F(t, x(t)),
\end{aligned}
$$

by (79) it is clear that the conclusions above include the results in Şenel [13].

Remark 13. When $0<\gamma<1$, if $g(t)=t$, the conclusions above are not applicable. Similarly, the assumption that $g(t) \geq$ $t$ in Şenel [12] and Qiu and Wang [10] should be changed to (C3) in this paper.

\section{Examples}

In this section, the application of our oscillation criteria will be shown in two examples. The first example is given to demonstrate Theorem 6 .

Example 1. Consider the equation

$$
\begin{aligned}
& \left(t^{\lambda}\left(\frac{1}{t}\left(x^{\Delta}(t)\right)^{\gamma}\right)^{\Delta}\right)^{\Delta}+\frac{2+\sin \left(x^{\Delta}(t)\right)}{t^{2}} x^{\gamma}(\sigma(t)) \\
& +\frac{1+\left(x^{\Delta}(t)\right)^{2}}{t^{3}} x^{\gamma}(g(t))=0,
\end{aligned}
$$

where $r_{1}(t)=t^{\lambda}, \lambda$ is a constant and $0 \leq \lambda<1, r_{2}(t)=1 / t$, $\gamma>\lambda, g(t) \geq \sigma(t), t_{0}=1$, and $t \in[1, \infty)_{\mathbb{T}}$, by (C4) we get $q(t)=1 / t^{3}$. Letting $H(t, s)=(t-s)^{2},(A, B)=\left(s^{3}, 0\right)$, we have

$$
\begin{gathered}
\int_{t_{0}}^{\infty} \frac{1}{r_{1}(t)} \Delta t=\int_{1}^{\infty} \frac{1}{t^{\lambda}} \Delta t=\infty \\
\int_{t_{0}}^{\infty} \frac{1}{r_{2}^{1 / \gamma}(t)} \Delta t=\int_{1}^{\infty} t^{1 / \gamma} \Delta t=\infty \\
\int_{t_{0}}^{\infty} q(t) \Delta t=\int_{1}^{\infty} \frac{1}{t^{3}} \Delta t<\infty \\
\delta(t)=\int_{t_{1}}^{t} \frac{\Delta s}{r_{1}(s)}=\int_{t_{1}}^{t} \frac{\Delta s}{s^{\lambda}}=O\left(t^{1-\lambda}\right) .
\end{gathered}
$$

Since $\gamma>\lambda$, when $\lambda<\gamma<1$,

$$
\begin{aligned}
\alpha(t) & =\left(\frac{2 \delta(t)}{r_{2}(t)} \int_{t}^{\infty} q(s) \Delta s\right)^{(1-\gamma) / \gamma} \\
& =\left(2 t \delta(t) \int_{t}^{\infty} \frac{1}{s^{3}} \Delta s\right)^{(1-\gamma) / \gamma}=O\left(t^{\lambda-\lambda / \gamma}\right),
\end{aligned}
$$

so we have

$$
\Phi_{1}(s)=\frac{r(s)\left(H_{2}^{\Delta}(t, s) A^{\sigma}(s)+H(t, s) A^{\Delta}(s)\right)^{2}}{4 \gamma H(t, s) A(s) \delta(s) \alpha(s)}=O\left(s^{1+\lambda / \gamma}\right),
$$


where $1+\lambda / \gamma<2$. Hence,

$$
\begin{gathered}
\limsup _{t \rightarrow \infty} \frac{1}{H\left(t, t_{1}\right)} \int_{t_{1}}^{t}\left[H(t, s)\left(2 A(s) q(s)-B^{\Delta}(s)\right)\right. \\
\left.-H_{2}^{\Delta}(t, s) B^{\sigma}(s)-\Phi_{1}(s)\right] \Delta s \\
=\limsup _{t \rightarrow \infty} \frac{1}{\left(t-t_{1}\right)^{2}} \int_{t_{1}}^{t}\left[2(t-s)^{2}-O\left(s^{1+\lambda / \gamma}\right)\right] \Delta s=\infty .
\end{gathered}
$$

When $\gamma \geq 1$,

$$
\begin{aligned}
\Phi_{1}(s)= & \frac{r_{2}(s)}{\delta(s)(H(t, s) A(s))^{\gamma}} \\
& \cdot\left(\frac{H_{2}^{\Delta}(t, s) A^{\sigma}(s)+H(t, s) A^{\Delta}(s)}{1+\gamma}\right)^{1+\gamma} \\
= & O\left(s^{2+\lambda-\gamma}\right),
\end{aligned}
$$

where $2+\lambda-\gamma<2$. Hence,

$$
\begin{gathered}
\limsup _{t \rightarrow \infty} \frac{1}{H\left(t, t_{1}\right)} \int_{t_{1}}^{t}\left[H(t, s)\left(2 A(s) q(s)-B^{\Delta}(s)\right)\right. \\
\left.-H_{2}^{\Delta}(t, s) B^{\sigma}(s)-\Phi_{1}(s)\right] \Delta s \\
=\limsup _{t \rightarrow \infty} \frac{1}{\left(t-t_{1}\right)^{2}} \int_{t_{1}}^{t}\left[2(t-s)^{2}-O\left(s^{2+\lambda-\gamma}\right)\right] \Delta s=\infty .
\end{gathered}
$$

That is, (50) holds. By Theorem 6 we see that (82) is oscillatory or $\lim _{t \rightarrow \infty} x(t)$ exists.

The second example illustrates Corollary 10.

Example 2. Consider the equation (see Şenel [13, Example $3.4]$ )

$$
\begin{array}{r}
x^{\Delta^{3}}(t)+\frac{a\left(1+\left(x^{\Delta}(t)\right)^{2}\right)}{t} x(t)+\frac{b}{t} x(t)=0, \\
a, b>0, \quad t \in[1, \infty)_{\mathbb{T}},
\end{array}
$$

where $r_{1}(t)=r_{2}(t)=1, \gamma=1, g(t)=t$, and $t_{0}=1$. Letting $c=\min \{a, b\}>0$, by (79) we have $q(t)=c / t$. Then, letting $(A, B)=(1,0)$, we obtain

$$
\begin{aligned}
& \int_{t_{0}}^{\infty} \frac{1}{r_{1}(t)} \Delta t=\int_{1}^{\infty} \Delta t=\infty \\
& \int_{t_{0}}^{\infty} \frac{1}{r_{2}^{1 / \gamma}(t)} \Delta t=\int_{1}^{\infty} \Delta t=\infty \\
& \int_{t_{0}}^{\infty} q(t) \Delta t=\int_{1}^{\infty} \frac{c}{t} \Delta t=\infty
\end{aligned}
$$

Therefore,

$\limsup _{t \rightarrow \infty} \int_{t_{1}}^{t}\left[2 A(s) q(s)-\Phi_{2}(s)\right] \Delta s=\limsup _{t \rightarrow \infty} \int_{t_{1}}^{t} \frac{2 c}{s} \Delta s=\infty$.
That is, (78) holds. By Corollary 10 we see that (89) is oscillatory or $\lim _{t \rightarrow \infty} x(t)=0$. This result is consistent with the conclusion in Şenel [13, Example 3.4].

\section{Conflict of Interests}

The author declares that there is no conflict of interests regarding the publication of this paper.

\section{Acknowledgment}

This project was supported by the NNSF of China (no. 11271379).

\section{References}

[1] S. Hilger, Ein Maßkettenkalkäul mit Anwendung auf Zentrumsmannigfaltigkeiten [Ph.D. thesis], Universität Wäurzburg, 1988.

[2] S. Hilger, "Analysis on measure chains-a unified approach to continuous and discrete calculus," Results in Mathematics, vol. 18, no. 1-2, pp. 18-56, 1990.

[3] R. P. Agarwal and M. Bohner, "Basic calculus on time scales and some of its applications," Results in Mathematics. Resultate der Mathematik, vol. 35, no. 1-2, pp. 3-22, 1999.

[4] R. Agarwal, M. Bohner, D. O’Regan, and A. Peterson, "Dynamic equations on time scales: a survey," Journal of Computational and Applied Mathematics, vol. 141, no. 1-2, pp. 1-26, 2002.

[5] M. Bohner and A. Peterson, Dynamic Equations on Time Scales: An Introduction with Applications, Birkhäuser, Boston, Mass, USA, 2001.

[6] M. Bohner and A. Peterson, Eds., Advances in Dynamic Equations on Time Scales, BirkhÄauser, Boston, Mass, USA, 2003.

[7] L. Erbe, T. S. Hassan, and A. Peterson, "Oscillation of third order nonlinear functional dynamic equations on time scales," Differential Equations and Dynamical Systems, vol. 18, no. 1-2, pp. 199227, 2010.

[8] T. S. Hassan, "Oscillation of third order nonlinear delay dynamic equations on time scales," Mathematical and Computer Modelling, vol. 49, no. 7-8, pp. 1573-1586, 2009.

[9] Y.-C. Qiu and Q.-R. Wang, "Kamenev-type oscillation criteria of second-order nonlinear dynamic equations on time scales," Discrete Dynamics in Nature and Society, vol. 2013, Article ID 315158, 12 pages, 2013.

[10] Y.-C. Qiu and Q.-R. Wang, "Oscillation criteria of second-order dynamic equations with damping on time scales," Abstract and Applied Analysis, vol. 2014, Article ID 964239, 11 pages, 2014.

[11] S. H. Saker, R. P. Agarwal, and D. O’Regan, "Oscillation of second-order damped dynamic equations on time scales," Journal of Mathematical Analysis and Applications, vol. 330, no. 2, pp. 1317-1337, 2007.

[12] M. T. Şenel, "Kamenev-type oscillation criteria for the secondorder nonlinear dynamic equations with damping on time scales," Abstract and Applied Analysis, vol. 2012, Article ID 253107, 18 pages, 2012.

[13] M. T. Şenel, "Behavior of solutions of a third-order dynamic equation on time scales," Journal of Inequalities and Applications, vol. 2013, article 47, 2013.

[14] Q. R. Wang, "Oscillation criteria for even order nonlinear damped differential equations," Acta Mathematica Hungarica, vol. 95, no. 3, pp. 169-178, 2002. 


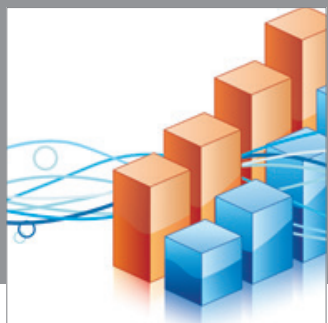

Advances in

Operations Research

mansans

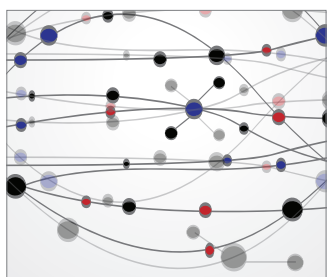

The Scientific World Journal
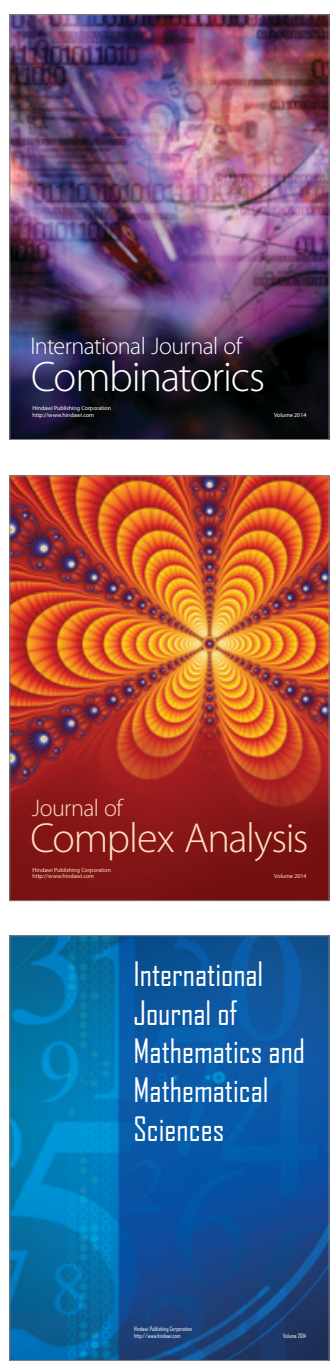
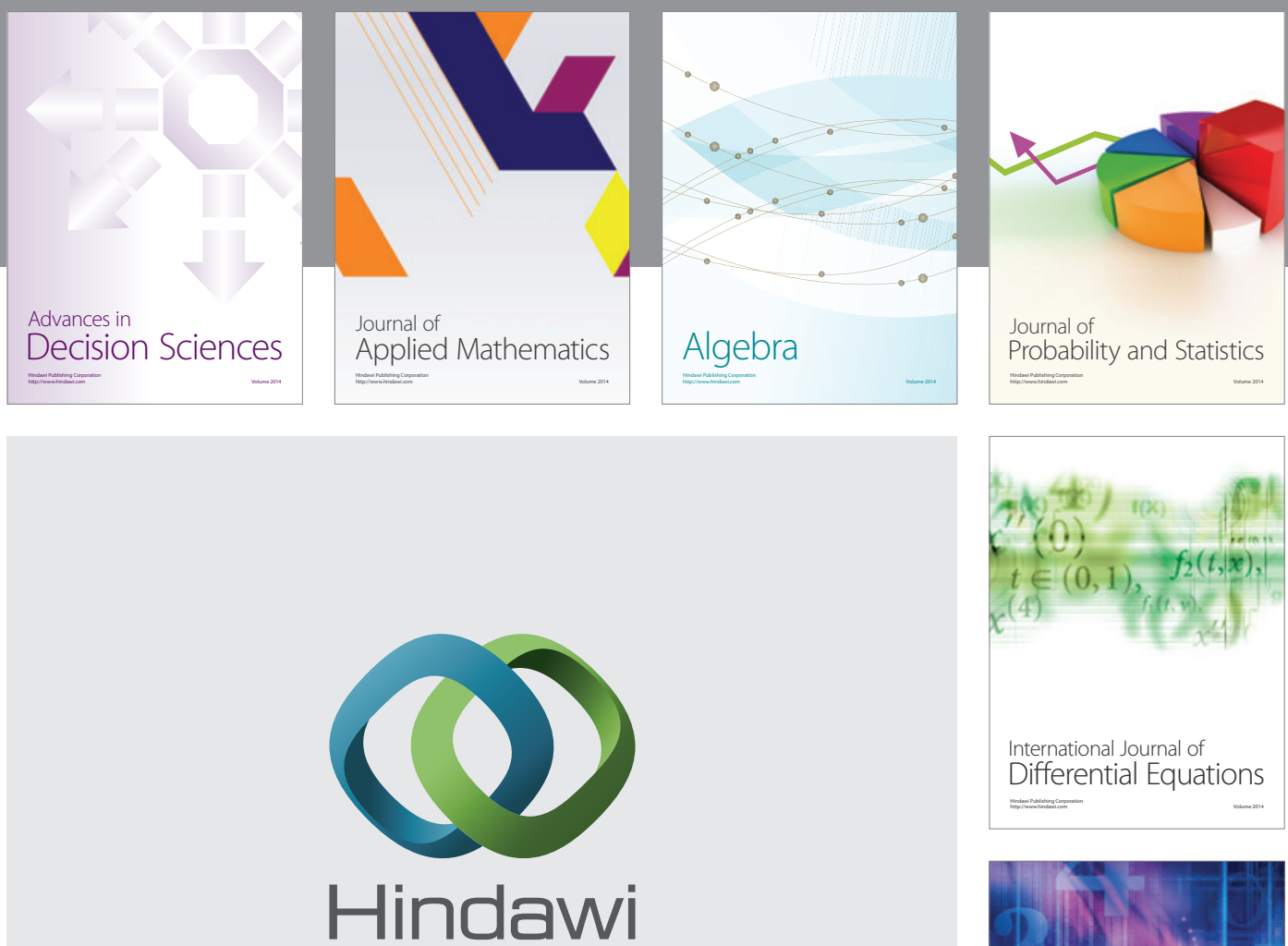

Submit your manuscripts at http://www.hindawi.com
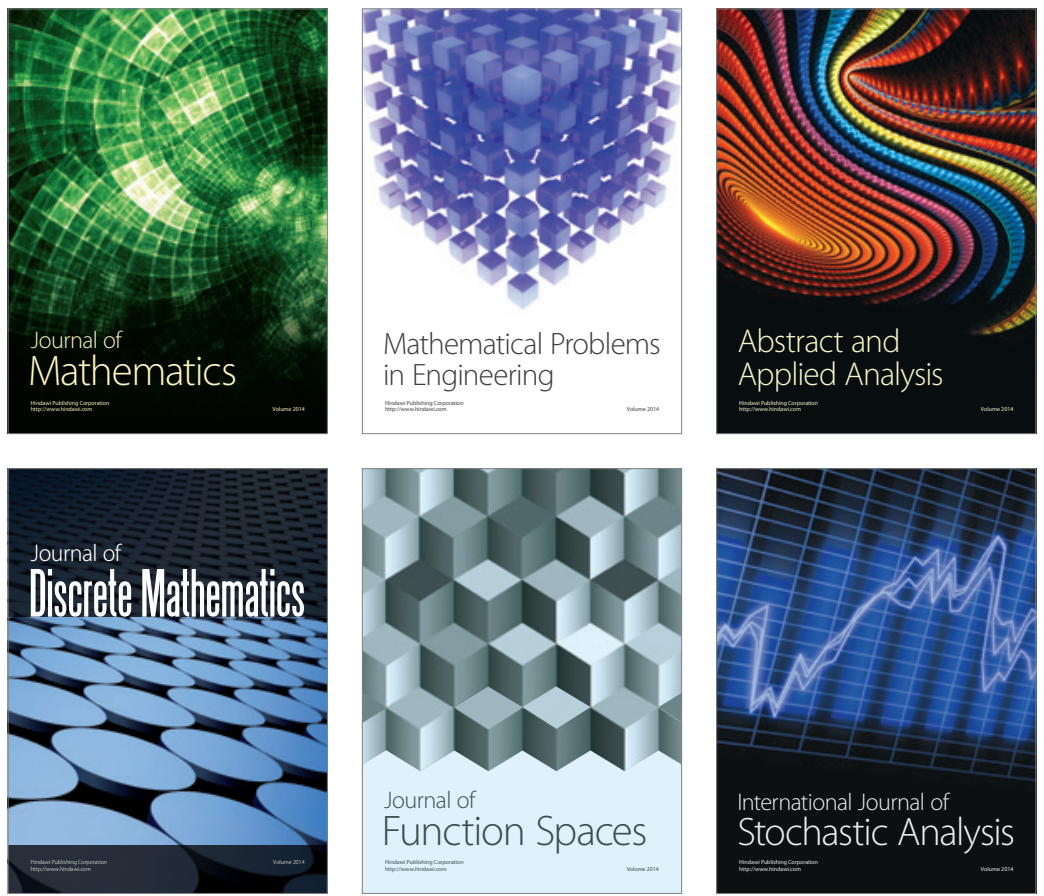

Journal of

Function Spaces

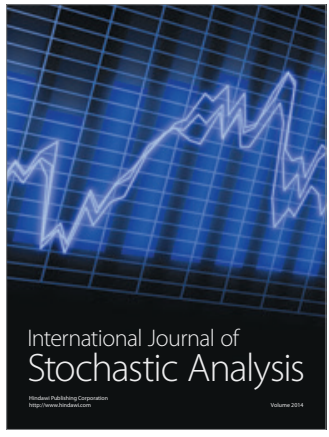

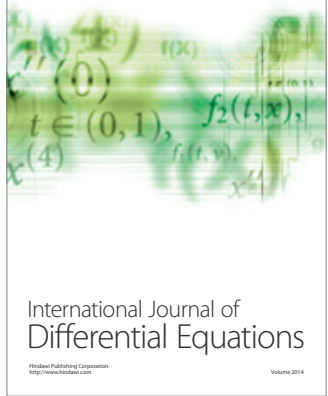
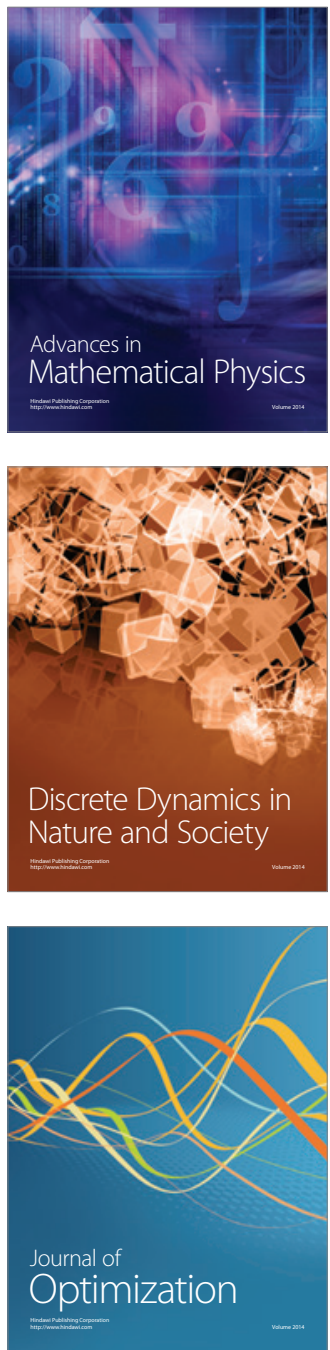